\title{
Effect of Bilateral Ultrasound-Guided Quadratus Lumborum Block versus Lumbar Epidural Block on Postoperative Analgesia following Major Lower Abdominal Cancer Surgery
}

\author{
Moaaz Mohamed Tohamy ${ }^{*}$, Samy Abdelrahman Amr ${ }^{1}$, Ashraf Amin Mohammed ${ }^{1}$, \\ Ahmad Mohamad Abd El-Rahman', Basma Rezk Farouk2, Mohamed Galal Mostafa El-Naggar3, \\ Mahmoud Mostafa Mohammed ${ }^{4}$, Montaser A. Mohamed1
}

\begin{abstract}
${ }^{1}$ Department of Anesthesia, Intensive Care and Pain Management, South Egypt Cancer Institute, Assiut University, Assiut, Egypt ${ }^{2}$ Department of Biostatistics and Cancer Epidemiology, South Egypt Cancer Institute, Assiut University, Assiut, Egypt

${ }^{3}$ Department of Clinical Pathology, South Egypt Cancer Institute, Assiut University, Assiut, Egypt

${ }^{4}$ Department of Surgical Oncology, South Egypt Cancer Institute, Assiut University, Assiut, Egypt

Email: ^moaaz670@gmail.com
\end{abstract}

How to cite this paper: Tohamy, M.M., Amr, S.A., Mohammed, A.A., El-Rahman, A.M.A., Farouk, B.R., El-Naggar, M.G.M., Mohammed, M.M. and Mohamed, M.A. (2021) Effect of Bilateral Ultrasound-Guided Quadratus Lumborum Block versus Lumbar Epidural Block on Postoperative Analgesia following Major Lower Abdominal Cancer Surgery. Open Journal of Anesthesiol$o g y, 11,335-346$.

https://doi.org/10.4236/ojanes.2021.1112033

Received: November 2, 2021

Accepted: December 11, 2021

Published: December 14, 2021

Copyright $\odot 2021$ by author(s) and Scientific Research Publishing Inc. This work is licensed under the Creative Commons Attribution International License (CC BY 4.0).

http://creativecommons.org/licenses/by/4.0/ (c) (i) Open Access

\begin{abstract}
Background: Poor postoperative pain control leads to longer postoperative care, longer hospital stay and decreased patient overall satisfaction. Aim: To compare the efficacy and safety of bilateral ultrasound-guided quadratus lumborum block versus lumbar epidural block on the management of postoperative pain following major lower abdominal cancer surgery. Methods: The study was a double-blinded, and randomized study, conducted in South Egypt Cancer Institute, Assiut University, Egypt. It included cancer patients scheduled for major lower abdominal cancer surgery in the period from 2019 to 2020 . They were divided into two groups: Group I received pre-emptive ultrasoundguided Quadratus Lumborum Block (QLB) with $25 \mathrm{~mL}$ of $0.25 \%$ bupivacaine on each side of the abdominal wall before induction of General Anesthesia (GA), and Group II received pre-emptive lumbar epidural block with $15 \mathrm{~mL}$ of $0.25 \%$ bupivacaine before induction of GA. VAS score, and time of the first analgesic request and postoperative total analgesic consumption were evaluated. Results: Sixty patients were included in our study. VAS score at rest was comparable between both studied groups in the first $6 \mathrm{~h}$. At 8 and $10 \mathrm{~h}$, Group II had a significantly higher VAS score at rest $(\mathrm{P}<0.001$ and 0.026 respectively). Meanwhile, at $12 \mathrm{~h}$, patients in Group I had a significantly higher VAS score $(\mathrm{P}=0.026)$. Mean time of the first request for rescue analgesia was significantly prolonged in Group I (13.27 $\pm 2.38 \mathrm{hrs}$.) compared to Group II $(10.20 \pm 1.42 \mathrm{hrs}).(\mathrm{P}<0.001)$ respectively, mean total morphine consumption,
\end{abstract}


over the first 24 hours postoperatively, was significantly lower in Group I $(5.17 \pm 1.32 \mathrm{mg})$ than in Group II $(7.33 \pm 1.45 \mathrm{mg})(\mathrm{P}<0.001)$. A larger number of patients in Group II had nausea at different time points postoperatively than in Group I $(\mathrm{P}<0.001)$, but no significant difference was observed between both studied groups regarding the incidence of vomiting. Limitation: Small sample size and shorter period for postoperative follow-up. Conclusions: Management of postoperative pain following major lower abdominal cancer surgery with US-guided QLB was associated with the reduction in the total analgesic consumption and delayed the first request of analgesia as compared to lumbar epidural block technique.

\section{Keywords}

Quadratus Lumborum Block, Lumbar Epidural Block, Postoperative Pain, Abdominal Cancer Surgery

\section{Introduction}

One of the main concerns of patients presenting for major abdominal surgery and anesthesiologists who are involved in postoperative care is postoperative analgesia [1]. Poor postoperative pain control leads to longer postoperative care, longer hospital stay and decreased patient overall satisfaction [2].

Opioids remain the mainstay of postoperative pain relief but have their own side effects. Therefore, the use of a multimodal analgesic strategy is nowadays preferable in most clinical settings to minimize the need for opioids. The use of regional anesthetic techniques provides excellent analgesia, as their effect can extend beyond the perioperative period [3].

Regarding major lower abdominal surgery, epidural analgesia was shown to be the gold standard time-tested technique for providing postoperative analgesia, but due to its possible complications, contraindications, there is a need for other equally efficient analgesic techniques [3].

Ultrasound-guided Quadratus Lumborum Block (QLB) is a relatively new regional anesthetic technique that was first described by Professor R. Blanco in 2007 at the annual scientific meeting of the European Society of Regional Anesthesia [4], it is relatively easily performed, the block effect lasts $24-48 \mathrm{~h}$ and up till now, no complications have been described during or after the block performance. QLB is safe and has found its place in multimodal postoperative pain strategy in patients undergoing abdominal surgery [5].

The aim of our study was to compare the efficacy and safety with regard to both techniques in the management of postoperative pain following major lower abdominal cancer surgery.

\section{Patients and Methods}

It was a double-blinded randomized study registered at

https://www.clinicaltrials.gov/ (NCT03958942). The study was conducted at South 
Egypt Cancer Institute, Assiut University, Egypt, and included all the patients who met the eligibility criteria in the period from 2019 to 2020. The protocol of the study was approved by Institutional Reviewing Board of South Egypt Cancer Institute.

\subsection{Sample Size}

This study enrolled all eligible cancer patients scheduled for major lower abdominal cancer surgery in the period from the first of January 2019 to the end of December $2020(n=60)$.

\subsection{Eligible Participants}

We included patient's $\geq 18$ years old, weight 50 - $85 \mathrm{~kg}$ with ASA II, III, who were scheduled for major lower abdominal cancer surgery (radical cystectomy, and total abdominal hysterectomy). The exclusion criteria were patients with history of relevant drug allergy, coagulation disorders, opioid dependence, sepsis or local infection at or near the vicinity of the block site, patients with psychiatric illnesses that would interfere with perception and assessment of pain, and patients who refused to participate in the study. The participants were divided into two groups. Group I received pre-emptive ultrasound-guided quadratus lumborum block with $25 \mathrm{~mL}$ of $0.25 \%$ bupivacaine on each side of the abdominal wall before induction of GA. Group II received pre-emptive lumbar epidural block with $15 \mathrm{~mL}$ of $0.25 \%$ bupivacaine before induction of GA. The allocation sequence was generated by a random number table, and group allocation was concealed in sealed opaque envelopes that were not opened until patient consent had been obtained. The patients and the investigators providing postoperative care were blinded to group assignment.

\subsection{Enrollment and Follow up Schedule}

Written consent was obtained from all participants after explaining the nature of the study. Then, patients were subjected to detailed history that included demographic data and full general examination including weight and height measurements and vital signs (patients' heart rate, non-invasive arterial blood pressure, respiratory rate and oxygen saturation) were assessed at operation room and before the start of anesthesia and monitored repetitively for the first 24 postoperative hours. Upon arrival at the operating room, the patients were placed in the supine position and an 18-gauge catheter was inserted intravenously in the dorsum of the hand. Lactated ringer solution $10 \mathrm{ml} / \mathrm{kg}$ was infused intravenously over $10 \mathrm{mi}$ nutes before the initiation of the local block. Standard monitoring probes (electrocardiography, non-invasive blood pressure, oxygen saturation, and temperature) was applied.

All groups were received pre-emptive conscious sedation by IV administration of $2 \mathrm{mg}$ midazolam.

Ultrasound-guided quadratus lumborum block technique: 
Bilateral ultrasound-guided transmuscular (anterior) quadratus lumborum block was performed using a SonoSite M-TurboTM ${ }^{\circledR}$ (SonoSite, Inc., Bothell, WA, USA) ultrasound machine and a curved $2-5 \mathrm{MHz}$ probe with a protective plastic sheath. Each patient was placed in the lateral position. After sterile prepping and draping, the transducer was placed in a transverse orientation on the flank just cranial to the iliac crest, then slide posteriorly to obtain an image of the quadratus lumborum muscle bordered by the lateral edge of the L3/L4 transverse process medially, psoas major muscle anteriorly and the erector spinae muscle posteriorly. A (Sono Plex ${ }^{\circledR}$ Stim cannula (PAJUNK) $22 \mathrm{G} \times 100 \mathrm{~mm}$, Germany) echogenic needle was inserted in-plane with the ultrasound beam in a posterior-to-anterior direction through the quadratus lumborum muscle until the ventral fascia of the muscle penetrated. At this point, the needle directed toward the fascial plan between the quadratus lumborum and the Psoas muscle; the correct position of the needle tip was confirmed by injecting $3 \mathrm{~mL}$ of saline to separate the fascial layers at the lateral aspect of the quadratus lumborum muscle. Thereafter, the needle was advanced more posteriorly between the quadratus muscle and psoas major muscle; (anterior to the quadratus lumborum muscle, between the epimysium of the quadratus lumborum muscle and the anterior thoraco-lumbar fascia) another $3 \mathrm{~mL}$ of saline was injected. After negative aspiration, $25 \mathrm{~mL}$ of $0.25 \%$ bupivacaine was injected in the fascial plane between the quadratus lumborum and psoas major muscles. The patient was then positioned on the contra-lateral side to perform the block on the other side in the same fashion.

Twenty minutes after quadratus lumborum block, we evaluate the extent of sensory blockade by using pin prick test. The classic dermatomes have been used: T6, xiphoid process; T10, umbilicus; T12, pubic bone area; and L1, inguinal ligament. Results of pin prick test were compared with the contra-lateral side: $(0=$ no block, 1 = hypoesthesia and 2 = anesthesia).

\section{Lumbar epidural block technique:}

Under strict aseptic precautions, lumbar epidural was performed for patients in Group II using a 16-gauge Touhy epidural needle by a median approach. The T12 - L1 or L1 - L2 interspaces was chosen for the injection.

The epidural space identified by the loss of resistance technique. The catheter was advanced $4 \mathrm{~cm}$ cephalad. When the aspiration test results for blood and cerebrospinal fluid were negative, a test dose of $(3 \mathrm{~mL}) 2 \%$ lidocaine with 1: 200,000 adrenaline was given after the placement of the epidural catheter.

\section{Standard general anesthesia:}

Surgery was performed under general anesthesia. After pre-oxygenation, intravenous anesthesia induced with propofol $1-2 \mathrm{mg} / \mathrm{kg}$, fentanyl $1 \mu \mathrm{g} / \mathrm{kg}$ administered over one min. Tracheal intubation was performed after adequate neuromuscular blockade with cisatracurium $0.15 \mathrm{mg} / \mathrm{kg}$. Anesthesia was maintained by 1 1.5 MAC isoflurane in $50 \%$ oxygen/air mixture and $0.03 \mathrm{mg} / \mathrm{kg}$ cisatrcurium, with ventilation parameters that maintain normocapnia. Intra-operative data will include vital signs via standard monitoring and duration of anesthesia and surgery. 
At the end of surgery, a reversal of the muscle relaxant was done using neostigmine $(0.04 \mathrm{mg} / \mathrm{kg})$ and atropine $(0.01 \mathrm{mg} / \mathrm{kg})$. After extubation, all patients were transmitted to the post anesthesia care unit (PACU). Post-operative analgesia comprised PCA with an initial morphine bolus of $0.1 \mathrm{mg} / \mathrm{kg}$ once pain was expressed by the patient or if VAS $\geq 3$, followed by $1 \mathrm{mg}$ boluses with a lockout period of 10 minutes.

Postoperatively, the presence and severity of pain, nausea and vomiting were be assessed at 1, 2, 4, 6, 12, 16, 20 and 24 hours by an investigator who was blinded to group allocation (anesthesia resident). The severity of pain at rest was assessed using a $10-\mathrm{cm}$ Visual Analogue Scale $(0=$ no pain and $10=$ worst imaginable pain). VAS, time to first request of rescue analgesia, and postoperative PCA morphine consumption during the first 24 hours postoperatively was recorded. The severity of nausea will be assessed by a categorical scale $(0=$ none, $1=$ mild, $2=$ moderate, 3 = severe). Rescue antiemetic will be given with metoclopramide $10 \mathrm{mg}$ IV when patients complained of nausea (score 2,3 ) or vomiting.

\subsection{The Study Outcomes}

The primary end point was to comparing the effects of bilateral ultrasound-guided quadratus lumborum block versus lumbar epidural block on postoperative analgesia following major lower abdominal cancer surgery.

\subsection{Statistical Analysis}

The data was collected and entered into Microsoft Excel Database to be analyzed using the Statistical Package for Social Science (SPSS Inc., Chicago, version 22). Quantitative variables were described in the form of mean \pm standard deviation. Qualitative variables were described as number and percent. In order to compare normally distributed quantitative variables between two groups, Student $\mathrm{t}$-test was performed, Mann Whitney $U$ test was used for non-normally distributed quantitative variables. Qualitative variables were compared using $\chi^{2}$ test or Fisher's exact test when the expected frequency is less than 5 . P value $<0.05$ is considered significant.

\section{Results}

A total of 60 patients were enrolled in this study and were randomly divided into the Group I (Quadrates block group, $\mathrm{n}=30$ ) and Group II (Epidural block, $\mathrm{n}=$ 30). Both studied groups were comparable for all baseline socio-demographic data, for age ( $51.47 \pm 4.83$ vs $52.93 \pm 7.58, \mathrm{P}=0.375)$, weight (79.33 \pm 4.51 vs $78.77 \pm$ $2.74, \mathrm{P}=0.559)$, height $(166.70 \pm 1.51$ vs $166.03 \pm 2.77, \mathrm{P}=0.252)$, and gender composition [6 (20.0\%) vs 8 (26.7\%), P = 0.542 were males; 24 (80.0) vs 22 (73.3) were female] in Group I versus Group II respectively (Table 1).

There is no statistically significant difference between the two groups as regard the duration of surgery and anesthesia $(4.07 \pm 0.45$ vs $3.97 \pm 0.41$ and 5.07 \pm 0.45 vs $4.97 \pm 0.41, \mathrm{P}=0.368)$ for both groups respectively, and also the preoperative vital signs (heart rate, systolic $\&$ diastolic $B P, R R$ and $\mathrm{O}_{2}$ saturation) were 
comparable between both groups with no significant difference between them $(\mathrm{P}>$ 0.05) (Table 2).

No significant difference was observed between both studied groups in mean VAS score at the first $6 \mathrm{~h}(\mathrm{P}>0.05$, for all). Then, patients in Group II recorded higher VAS scores at two successive time points (at $8 \mathrm{~h}$ the mean VAS score was $1.37 \pm 0.49$ in Group I vs $2.30 \pm 1.21$ in Group II, $\mathrm{P}<0.001$ ) and (at $10 \mathrm{~h}$ the mean VAS score was $2.17 \pm 1.21$ in Group I vs $3.20 \pm 1.69$ in Group II, $\mathrm{P}=0.026$ ). On the other hand, patients in Group I recorded higher VAS score after 12 hours ( $3.13 \pm 1.52$ in Group I vs $2.33 \pm 1.69$ in Group II, $\mathrm{P}=0.026)$, then both groups were comparable till the end of the first $24 \mathrm{~h}$. Group I needed longer time till the first request of rescue analgesia was needed (13.27 \pm 2.38 vs $10.20 \pm 1.42)$ and less total postoperative analgesic consumption in first 24 hours ( $5.17 \pm 1.32$ vs 7.33 $\pm 1.45)$ as compared to Group II ( $\mathrm{P}<0.001$ for both) (Table 3$)$.

Larger number of patients in Group II suffered from nausea at different time points postoperatively than in Group I; 12 (40.0\%) in Group II vs 5 (16.7\%) in Group I $(\mathrm{P}=0.045)$, but no significant difference was observed between both studied groups regarding the incidence of vomiting (3 (10.0\%) vs 7 (23.3\%) in Group I and Group II respectively $(\mathrm{P}=0.166)$ (Table 4$)$.

Table 1. Demographic data of the study participants $(n=60)$.

\begin{tabular}{|c|c|c|c|}
\hline Variable name & Group I $(n=30)$ & Group II $(n=30)$ & $\mathrm{P}$-value \\
\hline Age (years) & $51.47 \pm 4.83$ & $52.93 \pm 7.58$ & 0.375 \\
\hline Weight (Kg) & $79.33 \pm 4.51$ & $78.77 \pm 2.74$ & 0.559 \\
\hline Height (Cm) & $166.70 \pm 1.51$ & $166.03 \pm 2.77$ & 0.252 \\
\hline \multicolumn{4}{|l|}{ Sex, n (\%) } \\
\hline - Male & $(20.0)$ & $(26.7)$ & 0.542 \\
\hline - Female & $(80.0)$ & $(73.3)$ & \\
\hline
\end{tabular}

Data are presented as mean $\pm \mathrm{SD}$, or number (\%), Significance defined by $\mathrm{P}<0.05$. Group I: Quadratus block; Group II: Epidural block.

Table 2. Duration of surgery and anesthesia and preoperative vital signs assessment of the study participants $(n=60)$.

\begin{tabular}{cccc}
\hline Variable name & Group I $(\mathbf{n}=\mathbf{3 0})$ & Group II (n=30) & P-value \\
\hline Duration of surgery (h) & $4.07 \pm 0.45$ & $3.97 \pm 0.41$ & 0.368 \\
Duration of anesthesia (h) & $5.07 \pm 0.45$ & $4.97 \pm 0.41$ & 0.368 \\
Heart rate (beats/min) & $81.90 \pm 6.67$ & $83.73 \pm 5.72$ & 0.352 \\
Systolic BP (mmhg) & $136.67 \pm 10.61$ & $139.17 \pm 11.82$ & 0.541 \\
Diastolic BP (mmhg) & $82.00 \pm 5.81$ & $82.33 \pm 4.30$ & 1 \\
RR (cycle/min) & $13.87 \pm 0.51$ & $14.00 \pm 0.0$ & 0.154 \\
SO $_{2}$ & $97.73 \pm 0.45$ & $97.90 \pm 0.31$ & 0.098 \\
\hline
\end{tabular}

Data are presented as mean \pm SD, Significance defined by $\mathrm{P}<0.05$. Group I: Quadratus block; Group II: Epidural block. 
Table 3. Postoperative assessment of VAS score and first need of analgesia and the total analgesia consumption in $24 \mathrm{H}$ by the study participants $(n=60)$.

\begin{tabular}{cccc}
\hline Vas score & Group I $(\mathbf{n}=\mathbf{3 0})$ & Group II $(\mathbf{n}=\mathbf{3 0})$ & P-value \\
\hline $\mathbf{1}$ hour & $1 \pm 0.0$ & $1 \pm 0.0$ & 1 \\
$\mathbf{2}$ hour & $1 \pm 0.0$ & $1 \pm 0.0$ & 1 \\
$\mathbf{4}$ hour & $1.07 \pm 0.25$ & $1.20 \pm 0.41$ & 0.132 \\
$\mathbf{6}$ hour & $1.30 \pm 0.47$ & $1.37 \pm 0.49$ & 0.587 \\
$\mathbf{8}$ hour & $1.37 \pm 0.49$ & $2.30 \pm 1.21$ & $\mathbf{0 . 0 0 0}$ \\
$\mathbf{1 0}$ hour & $2.17 \pm 1.21$ & $3.20 \pm 1.69$ & $\mathbf{0 . 0 2 6}$ \\
$\mathbf{1 2}$ hour & $3.13 \pm 1.52$ & $2.33 \pm 1.69$ & $\mathbf{0 . 0 2 6}$ \\
$\mathbf{1 6}$ hour & $2.33 \pm 1.69$ & $1.70 \pm 1.44$ & 0.123 \\
$\mathbf{2 0}$ hour & $1.03 \pm 0.18$ & $1.07 \pm 0.25$ & 0.557 \\
$\mathbf{2 4}$ hour & $1.23 \pm 0.43$ & $1.47 \pm 0.51$ & 0.060 \\
First need of analgesia $(\mathrm{h})$ & $13.27 \pm 2.38$ & $10.20 \pm 1.42$ & $\mathbf{0 . 0 0 0}$ \\
Total analgesia in $24 \mathrm{H}$ & $5.17 \pm 1.32$ & $7.33 \pm 1.45$ & $\mathbf{0 . 0 0 0 *}$ \\
\hline
\end{tabular}

Data are presented as mean $\pm \mathrm{SD}$, ${ }^{\star}$ Significance defined by $\mathrm{P}<0.05$. Group I: Quadratus block; Group II: Epidural block.

Table 4. Postoperative assessment of side effects $(n=60)$.

\begin{tabular}{cccccc}
\hline Side effects & \multicolumn{2}{c}{ Group I $(\mathrm{n}=30)$} & \multicolumn{2}{c}{ Group II $(\mathrm{n}=30)$} & P-value \\
\hline Nausea & 5 & $(16.7)$ & 12 & $(40.0)$ & $0.045^{*}$ \\
Vomiting & 3 & $(10.0)$ & 7 & $(23.3)$ & 0.166 \\
\hline
\end{tabular}

Data are presented as number $(\%),{ }^{*}$ Significance defined by $\mathrm{P}<0.05$. Group I: Quadratus block; Group II: Epidural block.

\section{Discussion}

There is increased need for adequate management of postoperative pain to avoid respiratory and cardiovascular complications that can arise due to inadequate analgesia. This has lead to increased need for using regional anesthetic techniques for adequate postoperative pain management [6] [7].

For postoperative pain management following major lower abdominal surgeries, a number of pain relief techniques can be used mainly US-guided quadratus lumborum block, epidural analgesia, other procedures include: TAP block, and infiltration of LA at the site of incision. Also QLB has been described as a corner stone for management of postoperative pain after abdominal surgery [8].

Epidural analgesia is shown to be the "gold standard" technique for providing excellent postoperative analgesia plus attenuation of neurogenic mediators of inflammation. But, it can disturb the hemodynamic profile of the patients especially in patients receiving anticoagulants therapy due to epidural catheter placement and removal [3].

In 2007, Blanco first introduced the US-guided QLB technique as a posterior 
extension of the TAP block [4]. It represents a more extensive abdominal analgesia by placing a curvilinear transducer in a transverse orientation slightly above the iliac crest in the posterior axillary line and uses the QL muscle as its basic sonographic landmark [9] [10].

The study demonstrates a better management of postoperative pain and more satisfaction among patients received US-guided QLB with reduction in the total analgesic consumption and delayed first request of analgesia as compared to those received epidural block.

Our results supported by the study of Kwak et al., 2020 in a randomized controlled study aimed to investigate the opioid-sparing effect of a unilateral QLB in laparoscopic nephrectomy on 60 patients undergoing laparoscopic nephrectomy. Patients were randomized into two groups as QLB and control group. QLB group received QLB with $25 \mathrm{~mL} 0.25 \%$ ropivacaine, and the control group received 25 $\mathrm{mL} 0.9 \%$ saline at anterolateral border of quadratus lumborum muscle preoperatively reported that, the postoperative pain at rest and on movement was significantly lower in the QLB group than in the placebo group up to the 24th postoperative hour $(\mathrm{P}<0.05)$, also in line with our results the author demonstrated that the cumulative postoperative opioid consumption was significantly lower in the QLB group than in the placebo group at $6 \mathrm{~h}, 24 \mathrm{~h}$, and $48 \mathrm{~h}$ after surgery $(\mathrm{P}<0.05)$, also Kwak et al., 2020 reported no significant differences in the incidence of postoperative nausea and vomiting, time to first flatus to measure the extent of paralytic ileus and the quality of recovery-15 (QoR-15) questionnaire among the studied groups [11], the study of Zhu et al., 2021 which aimed to explore the effect of QLB on postoperative cognitive function in elderly patients undergoing laparoscopic radical gastrectomy on 64 patients who were randomly divided into the QLB group (Q group, $\mathrm{n}=32$ ) and control group ( $\mathrm{C}$ group, $\mathrm{n}=32$ ). The author reported that, the VAS at rest was significantly lower in the $\mathrm{Q}$ group compared to the $\mathrm{C}$ group at all times after surgery $(\mathrm{P}<0.05)$ and the sufentanil consumption during the first $24 \mathrm{~h}$ postoperatively in the $\mathrm{Q}$ group were significantly lower than those in the $\mathrm{C}$ group $(\mathrm{P}<0.05)[12]$.

Also Wang et al., 2021 reported that, the postoperative VAS pain scores on coughing in the QLB group at $2 \mathrm{~h}, 6 \mathrm{~h}, 12 \mathrm{~h}, 24 \mathrm{~h}$ time points and VAS pain score at rest $2 \mathrm{~h}, 6 \mathrm{~h}$ after surgery were significantly lower in the QLB than in the Sham group $(\mathrm{P}<0.05)$, also patients in the QLB group had significantly less postoperative sufentanil consumption, compared with the Sham group $(\mathrm{P}<0.05)$ [13].

To the best of our knowledge, almost all previous publications have compared either one of our used techniques (QLB or epidural block) versus the application of only general anesthesia or other regional anesthetics technique.

In agreement with our results, the previous studies of Kuo et al., 2006 [14], Moawad and Mokbel, 2014 [15], Liu et al., 2019 [16], Kwak et al., 2020 [11], Wang et al., 2021 [13] and Zhu et al., 2021 [12] reported no significant difference in baseline characteristics and hemodynamic profile between the studied groups. 
Adequate postoperative pain control is a key therapeutic component of the complex multimodal rehabilitation program after major abdominal procedures [17]. In the present study no significant difference was observed between both studied groups in mean VAS score at the first $6 \mathrm{~h}$. Then, patients in Group II recorded higher VAS score at the two successive time points $(8$ and $10 \mathrm{~h})((\mathrm{P}<0.001)$ and 0.026) respectively. Meanwhile, patients in Group I recorded higher VAS score at a further time points $(12 \mathrm{~h})(\mathrm{P}=0.026)$. Following that, both groups' returns to record no significant difference in mean VAS score till the end of measurement points. Also Group I have longer time for first need of analgesia and less total analgesia consumption in first 24 hours as compared to Group I $(\mathrm{P}<0.001)$.

The application of opioids by epidural analgesia delivers the drug close enough to the spinal cord so that the opioids can inhibit pain transmission from afferent nerves to the central nervous system through interaction with pre- and post-synaptic opioid receptors in the dorsal horn [15]. Also QLB enhances the analgesic effect and provides visceral pain relief as a result of local anesthetics reaching the paravertebral region via the thoracolumbar fascia [18].

Our results come in-line with the study of Kuo et al., 2006 who reported that, VAS pain scores at rest, at 2 and $4 \mathrm{~h}$ after surgery, and during coughing at $12 \mathrm{~h}$ after surgery, were significantly lower in Groups IV lidocaine and TEA (thoracic epidural analgesia) compared with group $\mathrm{C}$ (control group) $(\mathrm{P}<0.001)$ [14]

Niraj et al., 2011 compared the analgesic efficacy of continuous posterior TAP analgesia and four-quadrant TAP block with epidural analgesia in patients scheduled for laparoscopic colorectal surgery and found statistically significant higher consumption of tramadol analgesia in the TAP group (400 mg) versus (200 $\mathrm{mg}$ ) in the epidural group $(\mathrm{P}=0.002)$, also the author report comparable pain scores between both studied groups with comparable incidence of PONV.

In line with our results, the study of Kadam et al., 2013 who studied the analgesic efficacy of continuous TAP with epidural analgesia for major abdominal surgery. The authors reported no significant differences in regard to pain scores at any point or over time, postoperative fentanyl requirement, and patient satisfaction [19].

Also our results were supported by the previous study of Moawad and Mokbel, 2014 who performs a prospective randomized study on one hundred patients (ASA I or II) scheduled for elective major abdominal surgery at Gastroenterology surgical center, Mansoura University. Patients were allocated randomly into two groups (fifty patients each) to receive: patient-controlled epidural analgesia with bupivacaine $0.125 \%$ and fentanyl (PCEA group), or patient controlled intravenous analgesia with fentanyl (PCIA group). The author reported that, throughout the observation period $(24 \mathrm{~h}$ ), patients in the PCEA group had significantly less pain score and they were more satisfied by their pain therapy when compared with patients in PCIA group [15].

Kandi et al., 2015 evaluating the efficacy of US-guided TAP block versus epidural analgesia in management of postoperative pain following lower abdominal surgery and found that, patients with TAP block have effective postoperative analge- 
sia in the first $24 \mathrm{~h}$ with longer analgesic free periods in the TAP group compared to the epidural group during the first $24 \mathrm{~h}$ postoperatively. Also there was a significant reduction in the number of patients needing $>200 \mu \mathrm{g} / \mathrm{kg}$ of morphine in the TAP group as compared to the epidural group [20]. Blanco et al., 2015 published the first study investigating the analgesic effect of QL block after Cesarean Delivery (CD), in which $0.2 \mathrm{~mL} / \mathrm{kg} 0.125 \%$ bupivacaine was injected on the postero-lateral border of the QL muscle. A significant reduction in the morphine consumption and Vas scores was found during 48 hours after QL block administration [21]. In a second trial by the same author aimed to compare QLB versus transversus abdominis plane block (TAB) for pain relief and its duration of action after $\mathrm{CD}$ in a randomized controlled trial on 76 patients scheduled for elective CD found a significantly superior effect of the QL block lasting from 6 to 48 hours. No significant differences in visual analog scale results were shown between the 2 groups at rest or with movement. Calculated total pain relief at rest and with movement were similar $(\mathrm{P}<0.001)$ in both groups [22].

Iyer et al., 2017 in a study aimed to compare the analgesic efficacy of epidural analgesia versus ultrasound-guided Transversus Abdominis Plane (TAP) block and found that, the analgesia was comparable between both studied groups only till the first $16 \mathrm{~h}$ beyond which the patients in the epidural group reported better VAS scores both at rest and during coughing, also the paracetamol requirement in both groups were comparable [3].

Also, our results were in line with the study of Krohg et al., 2018 who perform a randomized, double-blinded, controlled trial aimed to evaluate the efficacy of the QL block after cesarean delivery. The author reported that, the effective analgesic scores were significantly better in the QLB group compared with the placebo group both at rest $(P<0.001)$ and during coughing $(P<0.001)$. The same author reported that the cumulative ketobemidone consumption in 24 hours was reduced in the QLB group compared with the control group $(\mathrm{P}=0.04$; ratio of means $=0.60 ; 95 \%$ confidence interval, $0.37-0.97)[10]$.

Our results show that patients in Group II suffered from opioid side effects mainly nausea at different time points postoperatively than patients in Group I $(\mathrm{P}=0.000)$. Kuo et al., 2006 demonstrated that the incidence of nausea or vomiting associated with morphine were higher among patients in group $\mathrm{C}$ (the control group), it observed in four, five and nine patients in groups TEA, IV lidocaine and $\mathrm{C}$ groups, respectively $(\mathrm{P}<0.01)$ [14]. Also our results supported by the previous randomized controlled study of Liu et al., 2019 which aimed to investigate the effects of combined epidural and general anesthesia on gastric cancer patients undergoing tumor resection on 107 patients with early stage gastric cancer. All patients in the control group $(n=54)$ were treated with general anesthesia, while patients in the observation group $(n=53)$ were treated with combined epidural and general anesthesia. The author reported that the incidence of nausea and vomiting, as well as postoperative agitation were significantly lower in the observation group than those in the control group $(\mathrm{P}<0.05)[16]$.

On the other hand, Moawad and Mokbel 2014 reported no significant differ- 
ence in the incidence of postoperative nausea, vomiting, pruritus, shivering and respiratory complications between the PCEA group and PCIA group [15].

\section{Limitation}

The limitation is small sample size and a short period for postoperative follow-up.

\section{Conclusion}

For major lower abdominal cancer surgery, management of postoperative pain with US-guided QLB is associated with lower 24-h VAS scores, opioid consumption and postoperative nausea and vomiting in comparison to epidural analgesia.

\section{Conflicts of Interest}

The authors report no conflicts of interest.

\section{References}

[1] White, P.F. and Kehlet, H. (2010) Improving Postoperative Pain Management: What Are the Unresolved Issues? Anesthesiology, 112, 220-225. https://doi.org/10.1097/ALN.0b013e3181c6316e

[2] Tano, P.F., Apiribu, F., Tano, E.K., Mensah, A.B.B., Dzomeku, V.M. and Boateng, I. (2021) Predicting Factors that Determine Patients' Satisfaction with Post-Operative Pain Management Following Abdominal Surgeries at Komfo Anokye Teaching Hospital, Kumasi, Ghana. PloS ONE, 16, e0251979. https://doi.org/10.1371/journal.pone.0251979

[3] Iyer, S.S., Bavishi, H., Mohan, C.V. and Kaur, N. (2017) Comparison of Epidural Analgesia with Transversus Abdominis Plane Analgesia for Postoperative Pain Relief in Patients Undergoing Lower Abdominal Surgery: A Prospective Randomized Study. Anesthesia, Essays and Researches, 11, 670-675. https://doi.org/10.4103/0259-1162.206856

[4] Blanco, R. (2007) Tap Block under Ultrasound Guidance: The Description of a "No Pops" Technique. Regional Anesthesia and Pain Medicine, 32, 130.

[5] Akerman, M., Pejčić, N. and Veličković, I. (2018) A Review of the Quadratus Lumborum Block and ERAS. Frontiers in Medicine, 5, Article No. 44. https://doi.org/10.3389/fmed.2018.00044

[6] Horn, R. and Kramer, J. (2019) Postoperative Pain Control. StatPearls Publishing, Treasure Island.

[7] Garimella, V. and Cellini, C. (2013) Postoperative Pain Control. Clinics in Colon and Rectal Surgery, 26, 191-196. https://doi.org/10.1055/s-0033-1351138

[8] Ahmed, A., et al. (2019) Ultrasound-Guided Quadratus Lumborum Block for Postoperative Pain Control in Patients Undergoing Unilateral Inguinal Hernia Repair, a Comparative Study between Two Approaches. BMC Anesthesiology, 19, Article No. 184. https://doi.org/10.1186/s12871-019-0862-Z

[9] Chakraborty, A., Khemka, R. and Datta, T. (2016) Ultrasound-Guided Truncal Blocks: A New Frontier in Regional Anaesthesia. Indian Journal of Anaesthesia, 60, 703-711.

[10] Krohg, A., Ullensvang, K., Rosseland, L.A., Langesæter, E. and Sauter, A.R. (2018) The Analgesic Effect of Ultrasound-Guided Quadratus Lumborum Block after Cesarean Delivery: A Randomized Clinical Trial. Anesthesia \& Analgesia, 126, 559-565. https://doi.org/10.1213/ANE.0000000000002648 
[11] Kwak, K.H., Baek, S.I., Kim, J.K., Kim, T.H. and Yeo, J. (2020) Analgesic Effect of Ultrasound-Guided Preoperative Unilateral Lateral Quadratus Lumborum Block for Laparoscopic Nephrectomy: A Randomized, Double-Blinded, Controlled Trial. Journal of Pain Research, 13, 1647-1654. https://doi.org/10.2147/JPR.S257466

[12] Zhu, M., Qi, Y., He, H.J., Zhang, S. and Mei, Y.L. (2021) Effect of Quadratus Lumborum Block on Postoperative Cognitive Function in Elderly Patients Undergoing Laparoscopic Radical Gastrectomy: A Randomized Controlled Trial. BMC Geriatrics, 21, Article No. 238. https://doi.org/10.1186/s12877-021-02179-w

[13] Wang, D., et al. (2021) Ultrasound Guided Lateral Quadratus Lumborum Block Enhanced Recovery in Patients Undergoing Laparoscopic Colorectal Surgery. Advances in Medical Sciences, 66, 41-45. https://doi.org/10.1016/j.advms.2020.12.002

[14] Kuo, C., Jao, S., Chen, K., Wong, C., Yeh, C., Sheen, M. and Wu, C. (2006) Comparison of the Effects of Thoracic Epidural Analgesia and i.v. Infusion with Lidocaine on Cytokine Response, Postoperative Pain and Bowel Function in Patients Undergoing Colonic Surgery. British Journal of Anaesthesia, 97, 640-646. https://doi.org/10.1093/bja/ael217

[15] Moawad, H.E.S. and Mokbel, E.M. (2014) Postoperative Analgesia after Major Abdominal Surgery: Fentanyl-Bupivacaine Patient Controlled Epidural Analgesia versus Fentanyl Patient Controlled Intravenous Analgesia. Egyptian Journal of Anaesthesia, 30, 393-397. https://doi.org/10.1016/j.egja.2014.06.002

[16] Liu, W., et al. (2019) Effects of General Anesthesia with Combined Epidural Anesthesia on Inflammatory Response in Patients with Early-Stage Gastric Cancer Undergoing Tumor Resection. Experimental and Therapeutic Medicine, 17, 35-40. https://doi.org/10.3892/etm.2018.6898

[17] Cindea, J., et al. (2012) The Impact of Epidural Analgesia on Postoperative Outcome after Major Abdominal Surgery. In: Fyneface-Ogan, S., Ed., Epidural Analgesia: Current Views and Approaches, InTech, Croatia, 55-72.

[18] Xu, M., et al. (2020) Quadratus Lumborum Block for Postoperative Analgesia after Cesarean Delivery: A Systematic Review and Meta-Analysis. International Journal of Obstetric Anesthesia, 42, 87-98. https://doi.org/10.1016/j.ijoa.2020.02.005

[19] Kadam, V.R., et al. (2013) Epidural versus Continuous Transversus Abdominis Plane Catheter Technique for Postoperative Analgesia after Abdominal Surgery. Anaesthesia and Intensive Care, 41, 476-481. https://doi.org/10.1177/0310057X1304100407

[20] Kandi, Y. (2015) Efficacy of Ultrasound-Guided Transversus Abdominis Plane Block versus Epidural Analgesia in Pain Management Following Lower Abdominal Surgery. Ains Shams Journal of Anaesthesiology, 8, 653-657. https://doi.org/10.4103/1687-7934.172762

[21] Blanco, R., Ansari, T. and Girgis, E. (2015) Quadratus Lumborum Block for Postoperative Pain after Caesarean Section: A Randomised Controlled Trial. European Journal of Anaesthesiology, 32, 812-818. https://doi.org/10.1097/EJA.0000000000000299

[22] Blanco, R., et al. (2016) Quadratus Lumborum Block versus Transversus Abdominis Plane Block for Postoperative Pain after Cesarean Delivery: A Randomized Controlled Trial. Regional Anesthesia \& Pain Medicine, 41, 757-762. https://doi.org/10.1097/AAP.0000000000000495 